\title{
Gratiarum actio
}

Was ich vor Jahresfrist über die Rückkehr zu Petron geschrieben habe, bedarf keiner Wiederholung. ${ }^{1}$ Mit umso größerem Vergnügen knüpfe ich an die Danksagungen des zweiten Bandes an.

Erleichtert haben meine Arbeit drei exzellente Kommentare jüngeren Datums: der von Natalie BREITENSTEIN zu Sat. 1-15 (Berlin 2009), der Gesamtkommentar von Gareth SCHMELING und Aldo SETAIOLI (Oxford 2011),2 sowie Giulio VANNINIs großer Wurf zu Sat. 100-115 (Berlin 2010 ), der in jeder Hinsicht Maßstäbe setzt. ${ }^{3}$

Höchst hilfreich war auch das Füllhorn von Petroniana, Sonderdrucken wie Büchern, die in den vergangenen Jahren ihren Weg nach Berlin fanden. Gedankt sei dafür auch an dieser Stelle (in der Hoffnung, niemanden vergessen zu haben), Francesca Romana BERNO (Rom), John BODEL (Providence, Rhode Island), Alberto BorghinI (Turin), Marcos CARMignANI (Córdoba, Argentina), Leonardo Costantini (Freiburg), Andrea Cucchiarelli (Rom), Basil Dufallo (Ann Arbor, Michigan), Lowell EDMunds (New Brunswick, New Jersey), Thomas GÄRTNER (Köln), Peter GrossardT (Leipzig), Erik HaMer (East Hampton, New York), Gerlinde Huber-Rebenich (Bern), Mario Labate (Florenz), Paolo Monella (Palermo), Gareth SCHMELING (Gainesville, Florida), Aldo Setaioli (Florenz), Antonio Stramaglia (Bari) und Heather Woods (Minneapolis, Minnesota).

Als Segen erwies sich die philologisch präzise, und zugleich erfrischend lesbare Neuübersetzung der Satyrica von Niklas HolzBERG (München), die er mir freundlicherweise zukommen ließ. Gareth SCHMELING übersandte mir seinen exquisiten neuen Petron für die ,Loeb Classical Library'. Giulio VANNINI (Florenz) verdanke ich neben den Separata seiner Aufsätze v.a. seinen kongenialen Lustrum-Bericht, der unschätzbare Dienste geleistet hat. Mit den Publikationen ihrer rührigen Arbeitsgruppe zum römischen Roman an der Universidad de Buenos Aires versorgte mich

Cf. Bd. II, S. IX-X.

S. meine Besprechung, Gymnasium 120, 2013, 361-363.

3 S. meine Besprechung, Ancient Narrative 10, 2012, 133-140. 
großherzig Josefina NAGORE $(\uparrow 2014)$. Aus einer lebhaften Korrespondenz erwuchs allmählich die Idee zu einem gemeinsamen Satyrica-Seminar am Rio de la Plata. Non ita dis visum est ... Dankbar gedenke ich auch der letzten Besuche bei Konrad MÜLLER $(† 2015)$ in Spiegel bei Bern.

In archäologischen Fragen berieten mich Johanna FABRICIUS (Berlin), Christian KUNZE (Regensburg), Monika TRÜMPER-RITTER (Berlin), Ralf VON DEN HOFF (Freiburg) und Detlev WANNAGAT (Rostock), in historischen Angelegenheiten Ernst BALTRUSCH und Wilfried NIPPEL (beide Berlin). Licht ins Dunkel des antiken Hüttenwesens warf HansGeorg DETTMER (Museumsbergwerk Rammelsberg bei Goslar); in montanen Angelegenheiten erteilte Annina MÜHLEMANN vom Schweizer Alpen-Club in Bern kompetent Auskunft. Ein epigraphisches Rätsel löste Ulrike EHMIG (Berlin), ein alttestamentarisches Martin RÖSEL (Rostock). Einblick in unpublizierte Fahnen des Thesaurus gewährte dessen Generalredaktor, Michael HiLlen (München). Niklas HOLZBERG verdanke ich die willkommene Gelegenheit, die Einleitung dieses Bandes dem bajuwarischen Ableger der ,Petronian Society' vorzustellen.

Im Hause de Gruyter trugen Torben BEHM, André HORN, Benedikt KRÜGER, Katharina LEGUTKE und Florian RUPPENSTEIN Sorge um das Projekt, Petron'.

Stefano POLETTI (Freiburg i.Br.) stellte seine Dissertation zur Verfügung, die in keiner Bibliographie erscheint, und von deren Existenz ich erst kurz vor Abgabe meines Manuskripts erfuhr. Seine umfängliche Exegese und sein Zeilenkommentar zu BC 1-155 zählen zum Besten, was über dieses Gedicht geschrieben wurde.

In textkritischen Fragen konnte ich abermals auf Giulio VANNINIs kundigen Rat zählen, dessen neue Petronausgabe allmählich Gestalt gewinnt. Die Lektüre des gesamten Manuskripts haben Marcus DEUfERT (Leipzig), Widu-Wolfgang EHLERS (Karlsruhe) und Aldo SETAIOLI (Florenz) auf sich genommen. Vor wie vielen Versäumnissen und peinlichen Versehen ihr unbestechlicher Scharfblick mich bewahrt hat, wie oft ihre Hinweise dabei halfen, die Argumentation zu glätten oder zu schärfen, steht mir lebhaft vor Augen. Schwerlich wird man im Folgenden eine Seite finden, der die souveräne Kritik dieser vier Autoritäten nicht heilsam zugute kam.

Ihnen allen sei auch an dieser Stelle von Herzen gedankt.

Berlin, am 27. Februar 2021

Peter Habermehl 